\title{
Transient receptor potential melastatin 8 ion channel in macrophages modulates colitis through a balance-shift in TNF-alpha and interleukin-10 production
}

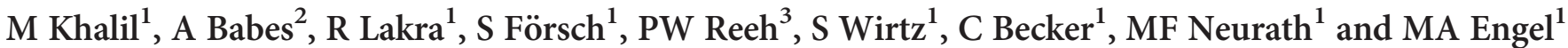

The transient receptor potential (TRP) ion channel family is well characterized in sensory neurons; however, little is known about its role in the immune system. Here we show that the cold-sensing TRPM8 has an unexpected role in innate immunity. TRPM8 expression and function in macrophages were demonstrated in vitro using molecular techniques and calcium imaging. In addition, adoptive macrophage transfer and systemic interleukin (IL)-10 overexpression were performed in experimental colitis. TRPM8 activation induced calcium-transients in murine peritoneal macrophages (PM) and bone marrow-derived macrophages of wild-type (WT) but not TRPM8-deficient mice. TRPM8-deficient PM exhibited defective phagocytosis and increased motility compared with those in WT, whereas the opposite effects of TRPM8 activation were induced in WT PM. TRPM8 activation or blockage/genetic deletion induced a anti- or proinflammatory macrophage cytokine profile, respectively. WT mice treated with repeated menthol (TRPM8 agonist) enemas were consistently protected from experimental colitis, whereas TRPM8-deficient mice showed increased colitis susceptibility. Adoptive transfer of TRPM8-deficient macrophages aggravated colitis, whereas systemic IL-10 overexpression rescued this phenotype. TRPM8 activation in peptidergic sensory neurons did not affect neuropeptide release from the inflamed colon. TRPM8 in macrophages determines pro- or anti-inflammatory actions by regulating tumor necrosis factor- $\alpha$ and interleukin-10 production. These findings suggest novel TRPM8-based options for immunomodulatory intervention.

\section{INTRODUCTION}

Macrophages are a principal element of innate immunity. The key functions of these cells are phagocytosis of invading microorganisms and environmental pathogens and regulation of the adaptive immune system. Macrophages are also critically involved in the regulation of gut homeostasis via the release of several cytokines and IL-10 receptor signaling. ${ }^{1}$ Little is known, however, about the role of the transient receptor potential (TRP) family of ion channels in the immune system, particularly in macrophages. TRP channels are integral membrane proteins with substantial calcium ion permeability. ${ }^{2}$ The TRP ion channel family comprises seven members, TRPC (canonical), TRPV (vanilloid), TRPP (polycystin), TRPML (mucolipin), TRPA (ankyrin), TRPN (NOMPC-like), and TRPM (melastatin), which are expressed in several tissues. ${ }^{2}$ Although TRP channels have been implicated in complex inflammatory pathologies such as colitis ${ }^{3-6}$ and asthma, ${ }^{7}$ the roles of many TRP channels are mainly described by their expression in sensory neurons or epithelial cells where they control neuropeptide or cytokine release, respectively. TRP channels have been widely recognized as fundamental for mediating specific responses to various physical or chemical noxious stimuli in sensory neurons. ${ }^{8}$ Through TRP receptors, sensory nerves can mediate defense mechanisms against

${ }^{1}$ Medizinische Klinik 1, Universitätsklinikum Erlangen, Erlangen, Germany. ${ }^{2}$ University of Bucharest Department of Physiology, Faculty of Biology, Bucharest, Romania and ${ }^{3}$ Institute of Physiology and Pathophysiology, Friedrich-Alexander-Universität Erlangen-Nürnberg, Erlangen, Germany. Correspondence: MA Engel (matthias.engel@uk-erlangen.de) 
damaging environmental and inflammatory stimuli to maintain the integrity of the organism ${ }^{8}$ - similar to the primordial function of the immune system.

Regarding macrophages, various TRP channels have already been shown to receive signals important for macrophage function. For example, TRPC3-deficient macrophages exhibited increased apoptosis and impaired efferocytosis, ${ }^{9}$ and TRPC6 upregulation was observed in human alveolar macrophages from patients with chronic obstructive pulmonary disease. ${ }^{10}$ In murine peritoneal macrophages (PM), TRPV2 mediated particle binding and phagocytosis was involved in lipopolysaccharide (LPS)-induced cytokine production, whereas TRPV $4^{+1+}$ macrophages restored the susceptibility of TRPV4 $^{-/-}$lungs to ventilator-induced mechanical injury. ${ }^{11,12}$

Little is known about TRPM8 expression in macrophages. TRPM8 (formerly known as Trp-p8 or CMR1) was previously characterized as a prostate cancer tumor marker ${ }^{13}$ and identified as an essential cold sensor in peripheral sensory neurons. ${ }^{14,15}$ Intriguingly, inflamed/injured sites have been treated via cooling likely for millennia. Ingredients derived from the mint plant family such as menthol or eucalyptol $(1,8$ cineol) are natural TRPM8 agonists and have been used for centuries to treat different inflammatory conditions. ${ }^{16}$ Moreover, menthol has recently been shown to inhibit cytokine production in human blood monocytes and lymphocytes in vitro. ${ }^{17}$ One recent report identified TRPM8-like channels in the RAW 264.7 macrophage cell line via patch-clamp analysis. ${ }^{18}$ Accordingly, we sought to determine whether TRPM8 has a functional role in macrophages and whether TRPM8 activation modulates inflammatory responses in vivo. Upregulated TRPM8 expression has recently been demonstrated under inflammatory conditions in both inflamed colonic tissues from patients with Crohn's disease and in a murine experimental colitis model. In addition, TRPM8 activation was shown to attenuate experimental colitis through the inhibition of neuropeptide release from sensory neurons. ${ }^{5,19}$ In contrast, another recent report suggested that TRPM8 mediated the release of calcitonin gene-related peptide (CGRP) from peptidergic sensory neurons, and direct effects of CGRP on $\mathrm{CD}_{1}{ }^{+}$dendritic cells (DCs) have been suggested as protective against colitis. $^{3}$

Here, we demonstrate that the activation of constitutively expressed TRPM8 in murine macrophages induces an anti-inflammatory cytokine profile and enhances phagocytosis, whereas genetic deletion or pharmacological block of TRPM8 induces the opposite effects. Consistent with these findings, menthol enemas were protective in wild-type (WT) mice with experimental colitis independently of neuropeptide release modulation whereas TRPM8-deficient mice exhibited aggravated colitis. Mice reconstituted with TRPM8-deficient macrophages consistently exhibited increased colitis susceptibility, demonstrating a fundamental role of constitutive TRPM8 expression in innate immunity. The pro-inflammatory effects of TRPM8-deficient macrophages were found to depend on an imbalance of tumor necrosis factor $\alpha$ (TNF)- $\alpha$ and interleukin (IL)-10 production in vitro and in vivo.

\section{RESULTS}

Functional TRPM8 expression in macrophage populations The RAW 264.7 cell line, a macrophage-like, Abelson leukemia virus-transformed line, is known to possess the characteristics of macrophages. Previously, one electrophysiological patchclamp analysis demonstrated TRPM8-like currents in these cells. ${ }^{18}$ Here we demonstrate TRPM8 immunoreactivity in RAW cells (Figure 1a). To further analyze TRPM8 functions in other phagocyte populations, we searched for TRPM8 in murine PM and identified TRPM8 immunopositivity in PM from WT mice but not in mice in which trpm 8 had been globally disrupted (KO) (Figure 1b). We confirmed TRPM8 protein expression in murine $\mathrm{WT}$ but not $\mathrm{KO} \mathrm{PM}$ via western blot analysis (Figure 1c). Moreover, using quantitative PCR, we demonstrated upregulated (doubled) trpm8 expression in PM in response to LPS $\left(100 \mathrm{ng} \mathrm{ml}^{-1}\right)$ treatment (Supplementary Figure S1a, b online). The numbers of PM in the peritoneal lavage fluid were similar in WT and TRPM8 KO mice, and there were no significant differences in macrophage morphology or immunoreactivity (F4/80) between the two genotypes (Figure 1b, Supplementary Figure S2). In addition, we demonstrate TRPM8 and F4/80 co-expression in colonic lamina propria mononuclear cells (LPMC) of WT but not TRPM8 KO mice (Figure 1d). Consistent with the observed TRPM8 expression in murine PM, ratiometric measurements using the cytosolic calcium indicator Fura-2 indicated large and sustained responses in WT PM in response to the TRPM8 agonist menthol at both $100 \mu \mathrm{M}(112 / 236$ responsive cells, $47 \%)$ (Figure 1e and f) and $10 \mu \mathrm{M}(144 / 420$ responsive cells, 34\%) (Supplementary Figure S3a, b). At both concentrations, responses to menthol were lacking in TRPM8-deficient PM, indicating the selectivity of menthol for TRPM8 at the tested concentrations. In addition, menthol $(100 \mu \mathrm{M})$ induced calcium ion transients in bone marrow-derived macrophages (BMDM) from WT mice (200/284 responsive cells, 70\%) but not from TRPM8 KO mice (Supplementary Figure S3c, d).

\section{TRPM8 modulates cytokine expression in macrophages}

We next addressed the possible involvement of TRPM8 in the control of cytokine release from macrophages. In both WT and TRPM8-deficient PM, the basal levels of released TNF- $\alpha$ and IL-10 were below $30 \mathrm{pg} \mathrm{ml}^{-1}$ or the detection limit, respectively (not shown). In vitro, LPS-induced TNF- $\alpha$ release was increased in TRPM8 KO PM relative to WT PM (Figure 2a), whereas IL-10 release was attenuated (Figure $\mathbf{2 b}$ ), suggesting that TRPM8 contributes to an anti-inflammatory cytokine profile. Consistent with these findings, menthol $(100 \mu \mathrm{M})$ inhibited LPS-induced TNF- $\alpha$ release and augmented IL-10 release in WT but not in TRPM8-deficient PM (Figure 2a and b). In accordance with the TRPM8mediated attenuation of TNF- $\alpha$ release, an immunoblot analysis revealed the menthol-induced decrease in phosphorylation of p38, a MAPK family member involved in the upregulation of TNF- $\alpha$ in macrophages. ${ }^{20}$ This effect was blocked by the co-application of the selective TRPM8 antagonist AMTB $(\mathrm{A}, 10 \mu \mathrm{M})$ (Figure $2 \mathrm{c}$ and e) and was lacking in TRPM8 
a

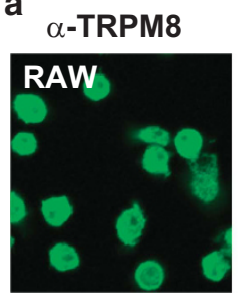

b
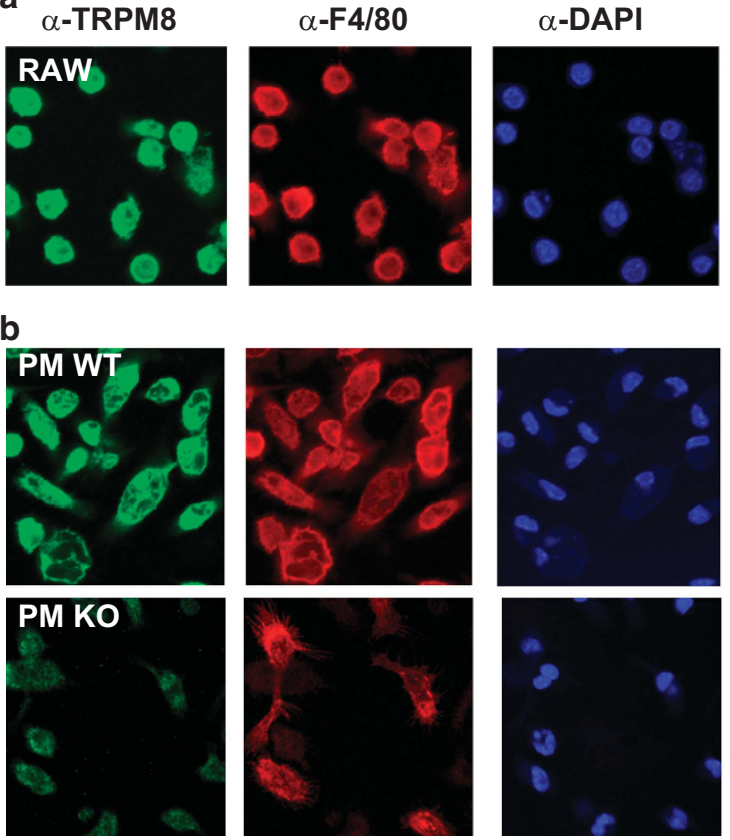
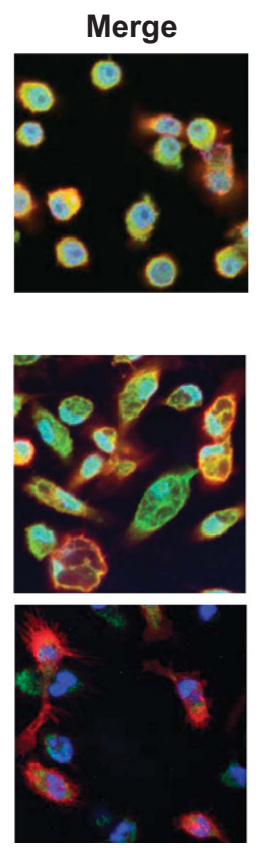
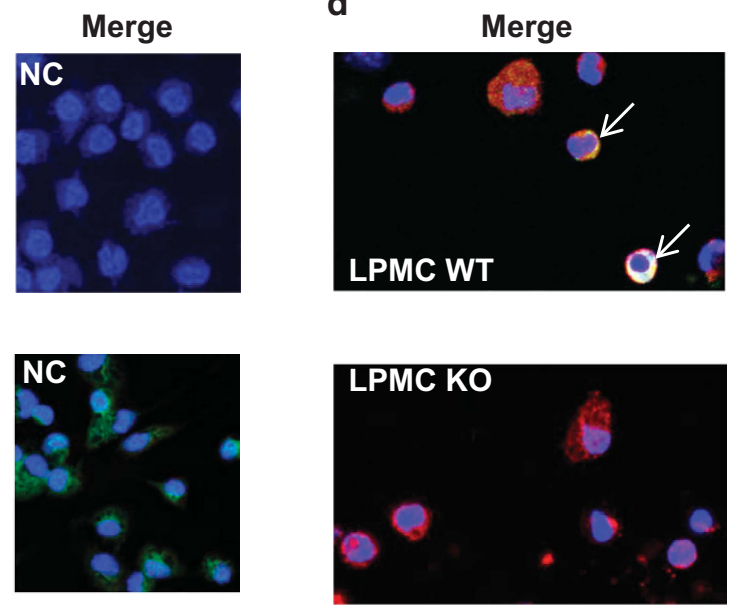

C kDa 130 WT KO

TRPM8 100

f
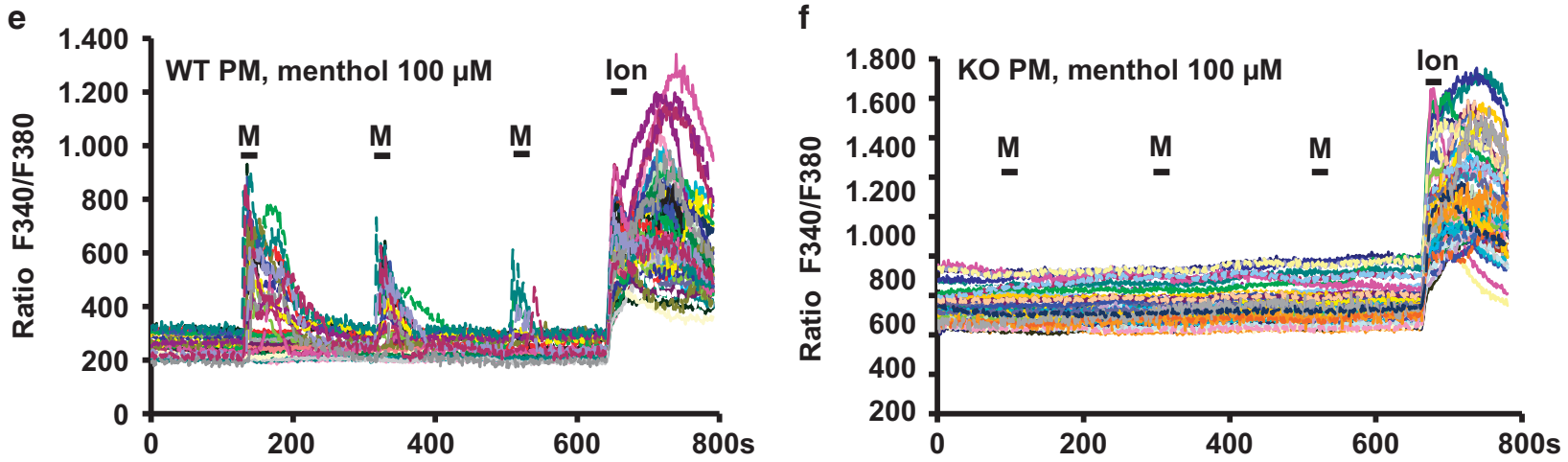

Figure 1 TRPM8 expression in different phagocyte populations. TRPM8 (green), F4/80 (red), and DAPI (blue) immunofluorescence staining ( $\alpha$-, anti-) in (a) RAW 264.7 macrophages and (b) peritoneal macrophages (PM) of wild-type (WT) and TRPM8-deficient (KO) mice with negative controls (NC). (c) Immunoblot analysis of WT and TRPM8-deficient peritoneal PM lysates with antibody to TRPM8. Recombinant mouse TRPM8 has a molecular mass of 128 kilodaltons $(\mathrm{kDa})$. (d) Co-expression of TRPM8 and F4/80 in colonic lamina propria mononuclear cells (LPMC) from WT (upper panel) and TRPM8 $\mathrm{KO}$ mice (lower panel). White arrows point at the yellow staining pattern as a result of TRPM8 (green) and F4/80 (red) immunofluorescence. (e,f) TRPM8mediated $\mathrm{Ca}^{2+}$ transients in PM. Representative Fura-2 traces of WT and KO PM stimulated with the TRPM8 agonist menthol (M, $\left.100 \mu \mathrm{M}\right)$ and ionomycin (Ion, $1 \mu \mathrm{M}$ ) presented as the ratio of emission after excitation at $340 \mathrm{~nm}$ to that after excitation at $380 \mathrm{~nm}$ (Ratio F340/F380). The lack of calcium responses in TRPM8-deficient macrophages indicates selectivity of menthol at TRPM8. Horizontal bars indicate stimulus periods.

KO PM (Figure 2d and e). Accordingly, menthol $(100 \mu \mathrm{M})$ enhanced the phosphorylation of extracellular-signal regulated kinase (ERK), a key regulator of IL-10 expression, ${ }^{21}$ in WT but not in TRPM8-deficient PM (Figure 2f and $\mathbf{g}$ ).

\section{TRPM8 modulates phagocytic activity and motility of macrophages}

To further explore the functional role of TRPM8, we determined its involvement in PM phagocytosis. Menthol $(100 \mu \mathrm{M})$-induced TRPM8 activation in vitro enhanced the uptake of zymosan particles, whereas this effect was reversed by co-application of the selective TRPM8 antagonist AMTB $(10 \mu \mathrm{M})$ (Figure 3a and b). Moreover, TRPM8-deficient PM isolated $1 \mathrm{~h}$ after the intraperitoneal (IP) injection of polystyrene microspheres in vivo exhibited impaired bead engulfment relative to WT PM (Figure $3 \mathbf{c}$ and d). Accordingly, the fraction of cells with high phagocytic activity (more than three bound/ingested particles) was reduced from $\sim 30 \%$ to $<10 \%$ (Figure 3d). In addition, bacterial phagocytic activity was also impaired in TRPM8-deficient PM exposed to Citrobacter rodentium in vitro for 3 and $6 \mathrm{~h}$ compared with WT PM (Figure 3e). The derived number of colony-forming units after lysing WT PM increased over time and was considerably higher than that achieved after lysing TRPM8deficient PM. Altogether, these findings suggest an immediate requirement for TRPM8 in the binding and phagocytosis of 
a

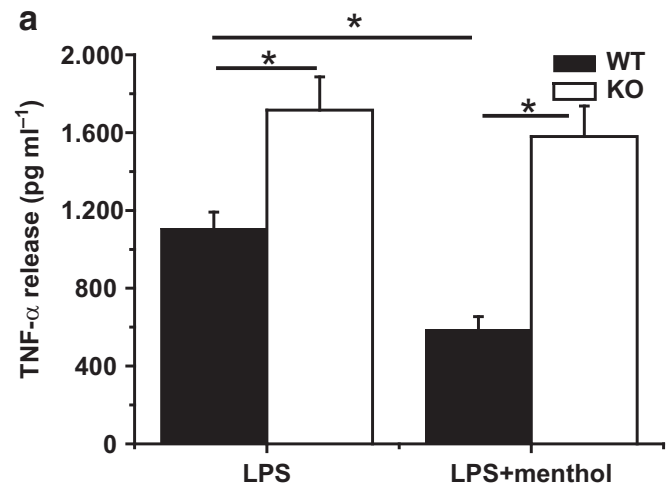

C

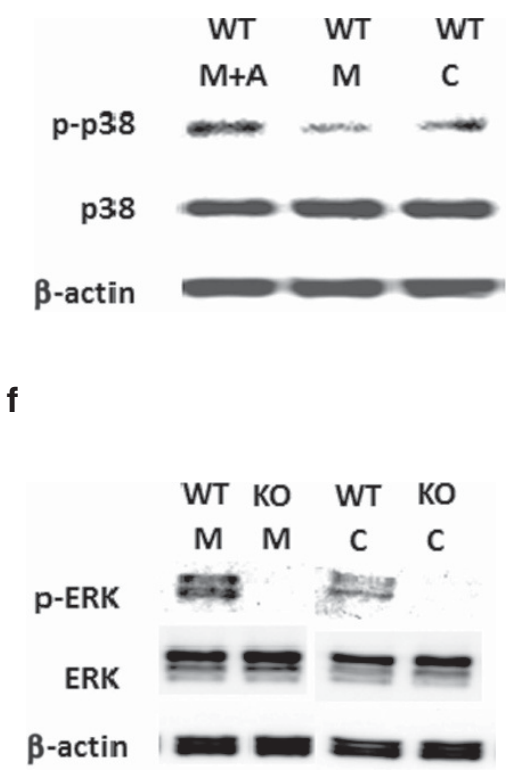

d

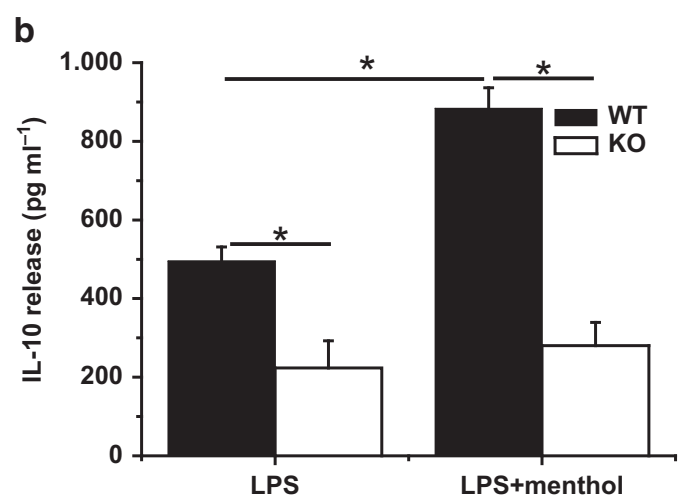

e

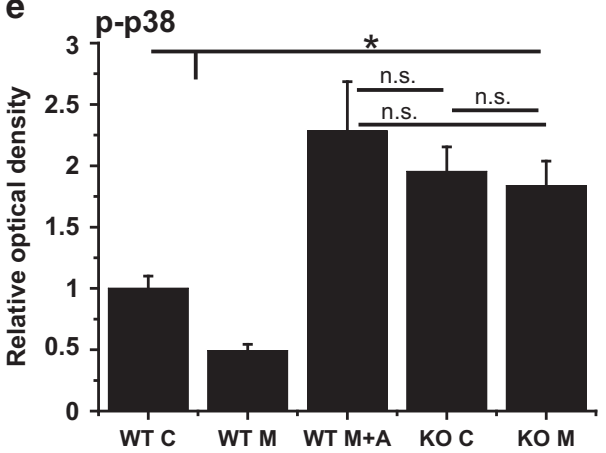

Figure 2 TRPM8-mediated modulation of cytokine expression in murine peritoneal macrophages (PM). Release of TNF- $\alpha$ (a) and IL-10 (b) from wild-type (WT) and TRPM8-deficient (KO) PM after $8 \mathrm{~h}$ of stimulation with LPS (100 $\left.\mathrm{ng} \mathrm{ml}^{-1}\right) \mathrm{w} /$ and w/o menthol (100 $\left.\mu \mathrm{M}\right)$. TRPM8 activation inhibits TNF- $\alpha$ but enhances IL-10 release in WT but not TRPM8-deficient PM. Data are from three experiments (mean and s.e.m. of four mice per genotype assayed in triplicate. ${ }^{*} P<0.05$, unpaired Student's $t$-test). (c) TRPM8-mediated downregulation of $p$-p38. Representative blot showing decreased immunofluorescence of p-p38 (42 kDa) in WT PM exposed to the TRPM8 agonist menthol (M, $100 \mu \mathrm{M})$ for $12 \mathrm{~h}$ compared with controls $(C$, vehicle). Coapplication of menthol and the TRPM8 antagonist AMTB (A, $10 \mu \mathrm{m})$ led to unchanged p-p38 expression compared with controls in the immunoblot analysis. (d) Representative blot showing decrease of p-p38 immunofluorescence in menthol (M, $100 \mu \mathrm{M})$-stimulated PM from WT but not KO mice compared with controls. (e) Quantitative analysis of the respective p-p38 western blot results showing the optical density (OD) of each band in relation to its $\beta$-actin OD and normalized to macrophages from WT control mice. Data are from three experiments (mean and s.e.m. of three mice each group. ${ }^{*} P<0.05$, unpaired Student's $t$-test). (f) TRPM8-mediated upregulation of $p$-ERK. Representative blot showing increased immunofluorescence of $p$-ERK (42 and $44 \mathrm{kDa}$ ) in WT PM exposed to the TRPM8 agonist menthol (M, $100 \mu \mathrm{m})$ compared with TRPM8 KO macrophages and controls (C, vehicle). (g) Quantitative analysis of the respective p-ERK western blot results showing the optical density (OD) of each band in relation to its $\beta$-actin OD and normalized to macrophages from WT control mice. Data are from three experiments (mean and s.e.m. of three mice each group. ${ }^{*} P<0.05$, unpaired Student's $t$-test).

different substrates. Moreover, compared with WT PM, the lack of TRPM8 elicited a stronger increase in the migration of starved PM (cultured in fetal calf serum, FCS $0.1 \%$ ) toward a high concentration of FCS (10\%) (Supplementary Figure S4a). Consistently, menthol-induced TRPM8 activation reduced cell migration, and this effect was blocked by the selective TRPM8 antagonist AMTB $(10 \mu \mathrm{M})$ (Supplementary Figure S4b).

\section{Role of TRPM8 in macrophages in the context of inflammation}

To explore the pathophysiological relevance of the above-described findings in the context of inflammation, we employed the DSS model of colitis, which is recognized as innate immune system-dependent. ${ }^{22} \mathrm{We}$ challenged one group of WT mice with twice-daily menthol $(100 \mu \mathrm{M})$ enemas to explore the possible therapeutic effect of topical TRPM8 

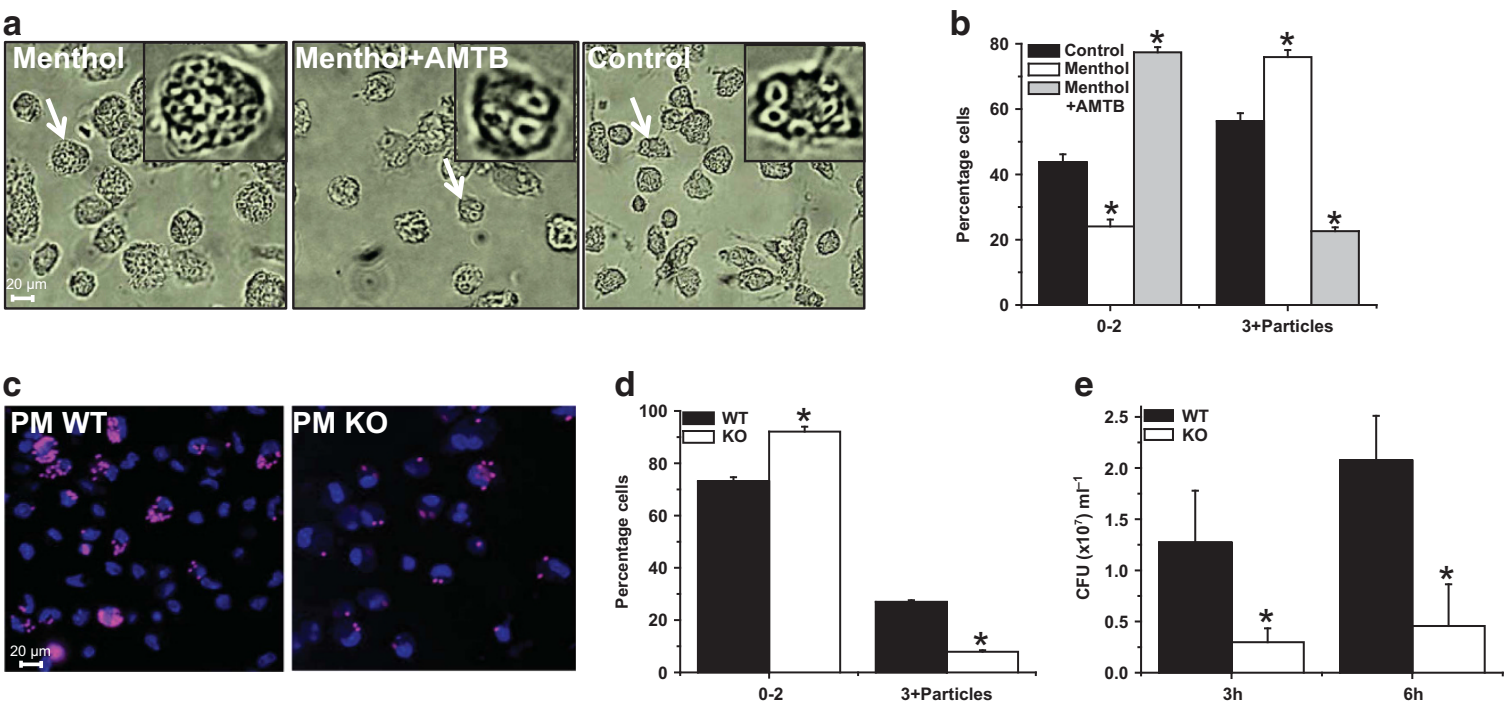

Figure 3 TRPM8-mediated modulation of phagocytosis in murine peritoneal macrophages (PM). (a) Representative pictures of wild-type (WT) PM under control conditions (control), w/ and w/o exposure to menthol $(100 \mu \mathrm{m})$ and/or the selective TRPM8 antagonist AMTB $(10 \mu \mathrm{m})$. (Scale bar, $20 \mu \mathrm{m}$; white arrow indicates cell that was magnified). (b) One hour exposure of PM to menthol $(100 \mu \mathrm{m})$ in vitro decreased the percentage of PM with a low number of ingested particles (0-2); however, increased the percentage of PM with a high number of ingested particles ( $3+$ ). Vice-versa, co-exposure of menthol and AMTB increased the percentage of PM with a low number of ingested particles (0-2); however, decreased the percentage of PM with a high number of ingested particles $(3+)$. (Data are representative of four experiments, ${ }^{*} P<0.05$, Mann-Whitney $U$-test). (c) Defective in vivo phagocytosis in TRPM8-deficient macrophages. Representative pictures of WT and TRPM8 KO PM isolated $1 \mathrm{~h}$ after IP injection of fluorescent beads (Scale bar, $20 \mu \mathrm{m}$ ). (d) In analogy to the in vitro results increased percentage of phagocytes with a low number (0-2) and decreased percentage of cells with a high number $(3+)$ of internalized polystyrene beads. (Data are representative of four experiments, ${ }^{*} P<0.05$, Mann-Whitney $U$-test). (e) Impaired bacterial internalization in TRPM8-deficient phagocytes. Internalization of Citrobacter rodentium after 3 and $6 \mathrm{~h}$ in PM from TRPM8 ${ }^{-1-}$ (KO) mice compared with wild-types (WT). Data are representative of three experiments (each four mice assayed in duplicate per genotype), ${ }^{\star} P<0.05$, Mann-Whitney $U$-test.

activation in dextrane sulphate sodium (DSS) colitis. Colitis severity was mild in the menthol enema group compared with control WT mice that received vehicle enemas (Figure 4). Colitis attenuation in menthol-treated mice was reflected by a little body weight loss (Figure 4a) compared with vehicle-treated control mice; furthermore, none of the mice treated with menthol enemas died during the course of DSS colitis (Figure 4b). Mini-endoscopic findings (day 7) were in accordance with the suggested protective effect of menthol enemas (Figure 4c); consistently, histological investigations demonstrated a reduced influx of inflammatory cells into the colonic wall with colonic architecture maintenance (Figure 4d, left panel), ultimately resulting in a distinctly reduced histopathologic score (Figure 4d, right panel).

To assess whether the protective effects of menthol enemas may involve the modulation of neuropeptide release as suggested previously, ${ }^{3,5}$ we next measured CGRP release from the isolated colons of healthy and colitic mice (Supplementary Figure S5). The TRPM8 agonists menthol $(100 \mu \mathrm{M})$ and icilin $(33 \mu \mathrm{M})$ inhibited mechanically-induced $(90 \mathrm{~mm} \mathrm{Hg})$ CGRP release in the healthy colon to approximately the same degree, both without exerting intrinsic effects on CGRP release. Interestingly, distension-induced colonic CGRP release from the inflamed colon (2\% DSS colitis) was strongly attenuated per se, suggesting depletion/desensitization of peptidergic sensory neurons. Neither TRPM8 agonists exhibited an effect on mechanically induced CGRP release under this condition.

To further explore the general role of TRPM8 in colonic inflammation, we induced DSS colitis in WT and TRPM8 KO mice. As determined by the same assessment tools as above, TRPM8 KO mice exhibited increased colitis susceptibility (Figure 5). Compared with control WT mice, TRPM8 KO mice lost more weight (Figure 5a), and a larger number of knockout mice died during the 7-day course of DSS colitis (Figure 5b). TRPM8 KO mice also exhibited greater endoscopic (Figure 5c) damage and more severe histological changes (Figure 5d) compared with control WT mice.

In an effort to dissect the role of TRPM8 in macrophage function in vivo, we used a clodronate liposome-induced macrophage depletion model. WT mice were repeatedly challenged with IP injections of clodronate liposomes, which induced a marked systemic reduction in F4/80-immunopositive cells in the colon, liver, and spleen, as demonstrated by immunocytochemical and flow cytometric measurements (Supplementary Figure S6a, b). Reconstituted macrophages had supposedly invaded the murine colon, as macrophage-depleted mice that were not reconstituted showed much less $\mathrm{F} 4 / 80+$ cells in the inflamed colon than controls (Supplementary Figure S6c). Macrophage-depleted mice were subsequently reconstituted intravenous (IV) with TRPM8 KO or WT BMDM that had been differentiated into macrophages in vitro via macrophage colony-stimulating factor stimulation 
a

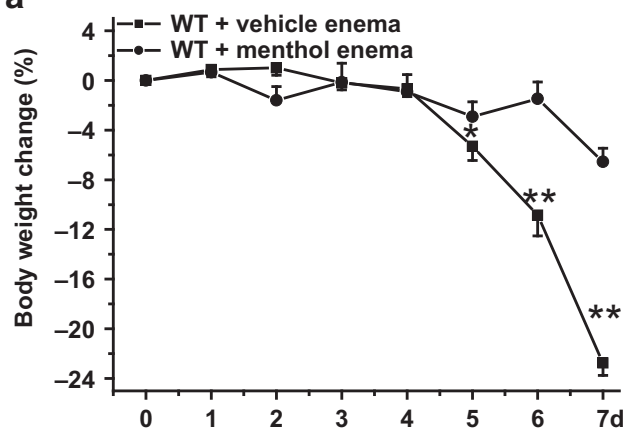

C

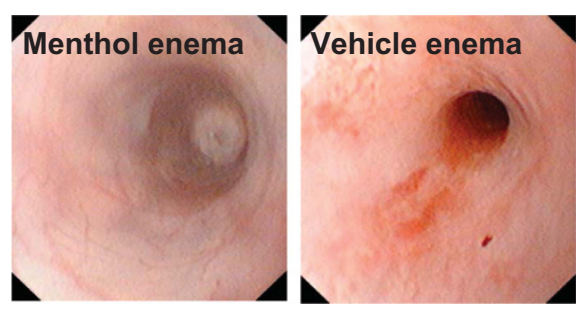

d

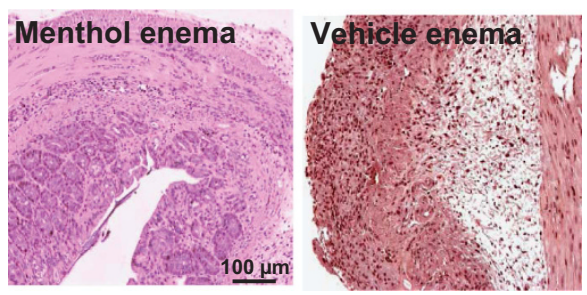

b
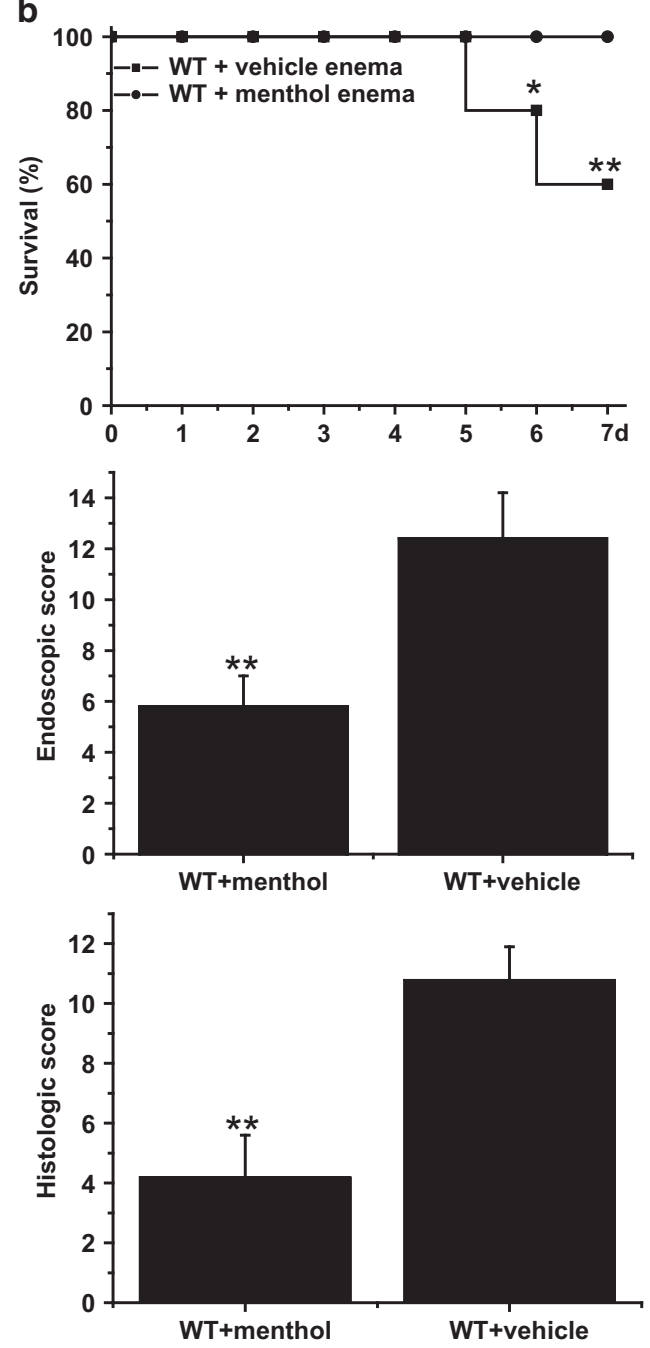

Figure 4 Menthol enemas protect wild-type WT C57BL/6 mice from DSS (2\%) colitis. (a) Mice that were treated with twice daily menthol (100 $\mu \mathrm{M})$ enemas only lost little weight during the course of DSS colitis compared with vehicle controls. Data are representative of three experiments, each group $n=6$. ${ }^{\star} P<0.05,{ }^{* \star} P<0.01$, analysis of variance followed by Fisher's LSD test. (b) Kaplan-Meier survival curve of the two groups. All mice that were treated with menthol enemas during the course of the DSS colitis stayed alive. (c) Representative endoscopical photographs of the colon from the two groups and respective endoscopical score (on day 7). (d) Representative pictures of H\&E stainings ( $\times 20$ magnification, scale bar 100 $\mu \mathrm{m})$ from the murine colon of the both groups. Menthol-treated WT mice showed a reduced histological score comparedwith controls. ${ }^{\star \star} P<0.01 \mathrm{Mann}-\mathrm{Whitney} U$-test.

according to an established protocol (see the Supplementary Methods section). A scheme with a detailed experimental protocol/timeline is given in Figure 6a. Mice were treated with $2 \%$ DSS in drinking water. Moderate colitis was observed in mice reconstituted with WT macrophages, whereas severe disease occurred in mice receiving $\mathrm{KO}$ macrophages. The colitis susceptibility in the latter group was reflected by increased body weight loss (Figure 6b) with poorer survival among mice receiving $\mathrm{KO}$ macrophages (Figure $\mathbf{6 c}$ ). Similar to the in vitro findings, the level of colonic IL-10 content was reduced in mice receiving $\mathrm{KO}$ macrophages (Figure 6d, left panel); however, the corresponding increase in colonic TNF- $\alpha$ levels did not reach statistical significance (Figure 6d, right panel). Endoscopic examination of the three groups (on day 6) confirmed signs of inflammation such as fibrin exudate, hemorrhagic lesions, and increased mucosal edema in the mice receiving $\mathrm{KO}$ macrophages compared with WT macrophages (Figure 6e). Reactive oxygen species (ROS) generation is inherent to innate immune responses. ROS are not only crucially involved in host defense against pathogens but also act as signaling agents to coordinate cytokine production. In vivo fluorescence imaging revealed that the concentration of ROS was distinctly higher in the colons of mice reconstituted with $\mathrm{KO}$ macrophages compared with WT macrophages (Figure 6f). Consistently, mice that received TRPM8-deficient macrophages exhibited a severe influx of inflammatory cells with profound colonic architecture damage, which was semi-quantified by a higher histopathological damage score (Figure 6g). In a separate control experiment the effect of macrophage depletion on colitis severity per se was also analyzed (Supplementary Figure S7). Clodronate liposome-treated macrophagedepleted WT mice per se showed DSS (2\%) colitis 

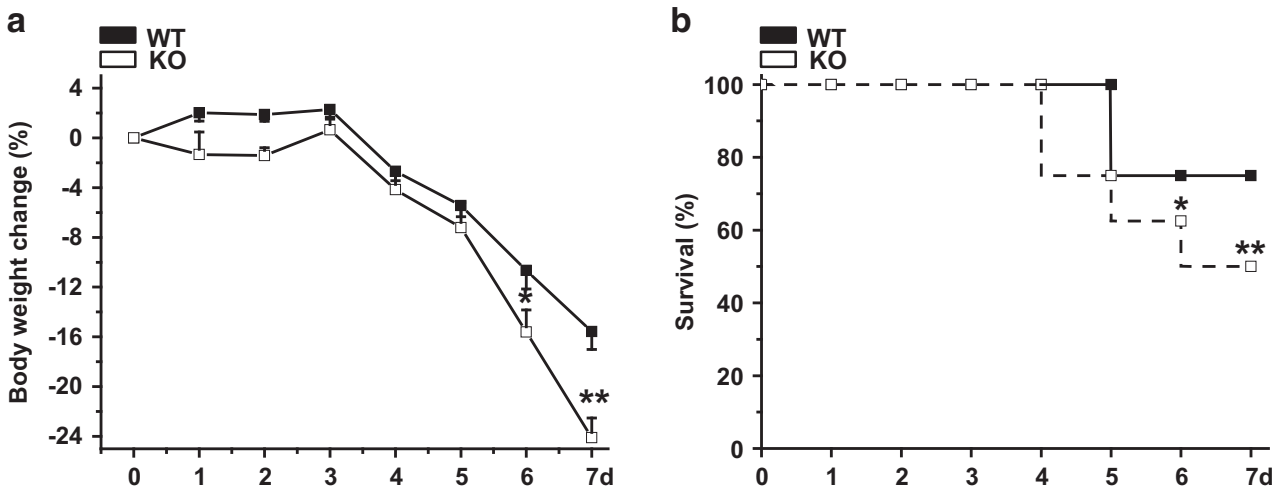

C
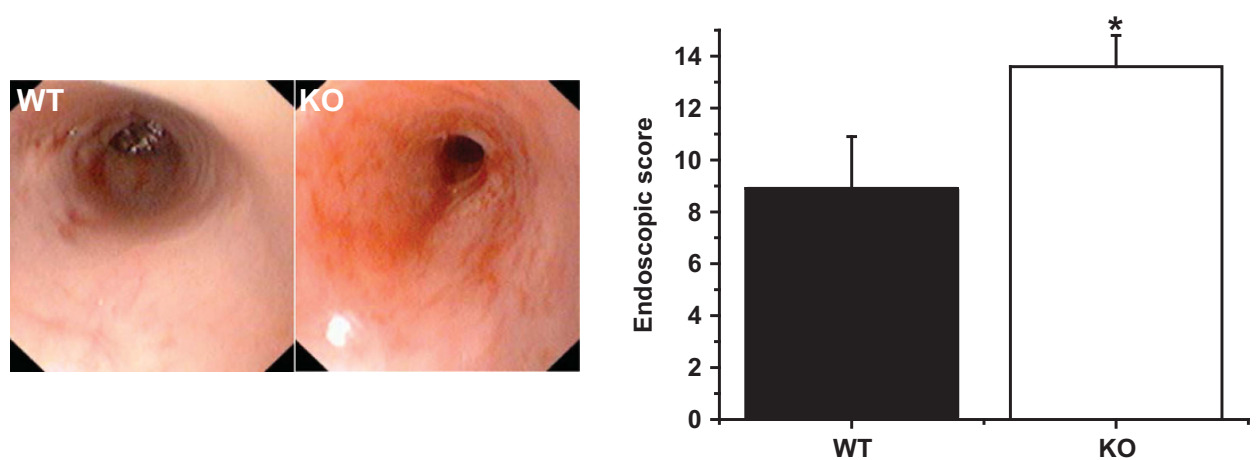

d
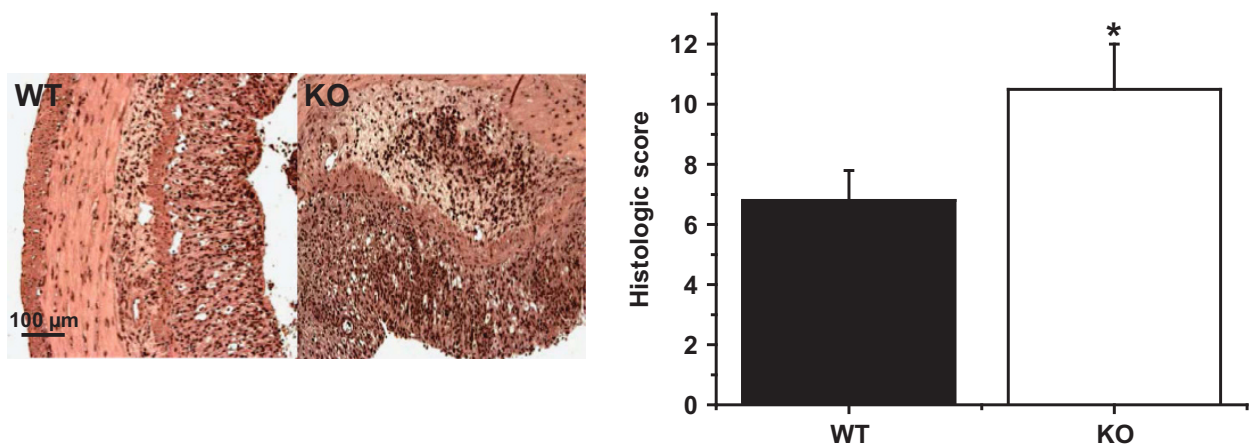

Figure 5 Aggravated DSS (2\%) colitis in TRPM8-deficient (KO) compared with wild-type (WT) mice. (a) Weight loss in TRPM8-deficient mice was more severe compared with control WT mice during the course of DSS colitis in this separate experiment. Data are representative of three experiments, each group $n=6$. ${ }^{\star} P<0.05,{ }^{* \star} P<0.01$ analysis of variance followed by Fisher's LSD test. (b) Higher letality in TRPM8-deficient compared with WT mice with DSS colitis in the Kaplan-Meier survival curve of the two groups. (c) Representative endoscopical photographs demonstrate increased macroscopic damage in the colon of TRPM8-deficient mice compared with control WT mice, which is mirrored in the increased semiquantitative endoscopic score. (d) Representative H\&E stainings ( $\times 20$ magnification, scale bar $100 \mu \mathrm{m}$ ) from the colon of WT and TRPM8-deficient mice and respective histologic scores. ${ }^{*} P<0.05$ Mann-Whitney U-test.

attenuation compared with mice that received vehicle PBS injections IP instead of clodronate liposomes, which emphasize the important role of macrophages in DSS colitis development.

To determine whether the lack of IL-10 production in TRPM8-deficient macrophages was responsible for increased colitis susceptibility we overexpressed bioactive IL-10 protein in vivo using IL-10-encoding DNA minicircles. Notably, IL-10 overexpression rescued the colitis susceptibility in mice receiving TRPM8-deficient macrophages as demonstrated by (Figure 7a) the body weight course (Figure 7b) survival rate, as well as the endoscopical (Figure 7d) and histological picture (Figure 7e). Colonic IL-10 levels were reduced in mice with DSS colitis that were reconstituted with TRPM8-deficient macrophages and elevated in those overexpressing IL-10 compared with mock-treated mice reconstituted with $\mathrm{TRPM}^{+/+}$macrophages (Figure 7c, left panel). IL-10 overxpression in mice that were reconstituted with WT macrophages significantly attenuated DSS colitis. These results suggest that a lack of immunoregulatory IL-10 is responsible for the aggressive/pro-inflammatory phenotype of TRPM8-deficient macrophages in vivo. To demonstrate that WT macrophages are the vital target of the menthol enemas we performed another experiment. There, global TRPM8 KO mice were depleted and reconstituted with WT macrophages. This 
group was compared with various control groups (Figure 8a-d). Only mice that harbored WT macrophages were protected from DSS colitis by menthol enemas independent of TRPM8 expression in non-macrophage tissue.

\section{DISCUSSION}

For the first time, we have provided evidence for the functional expression of TRPM8 in various types of macrophages and demonstrated its role as a key modulator of macrophage

a

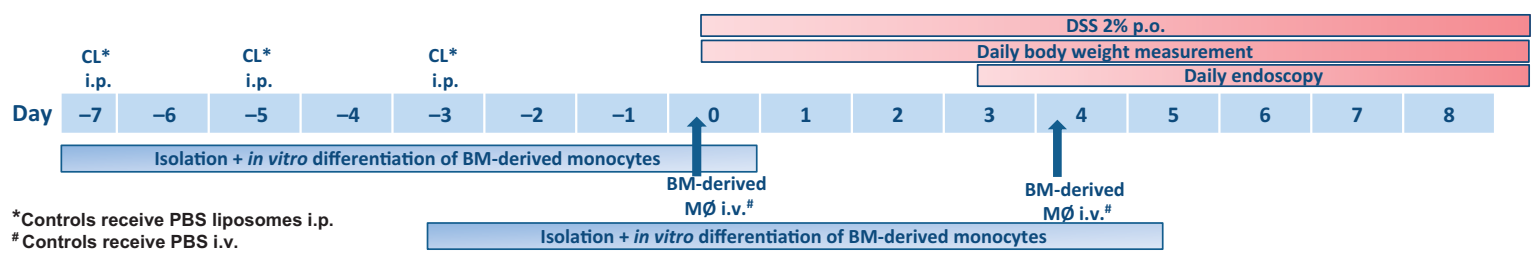

b

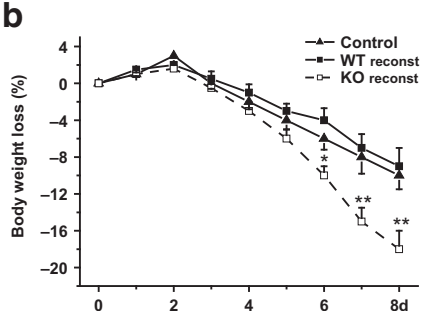

C
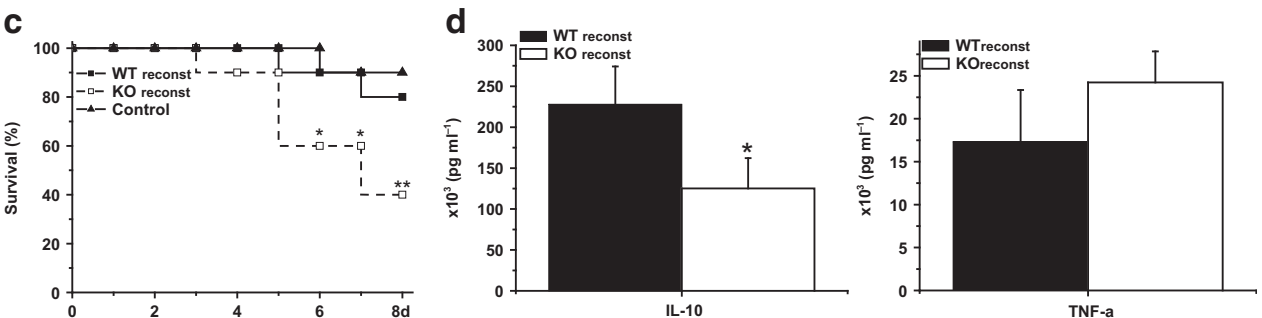

e
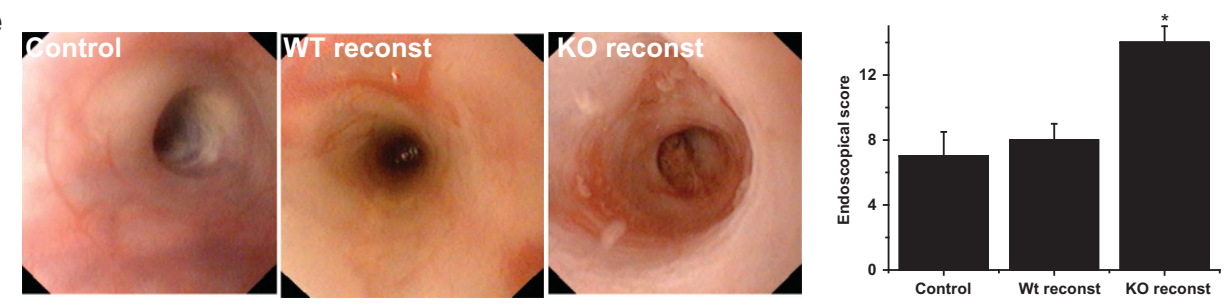

f

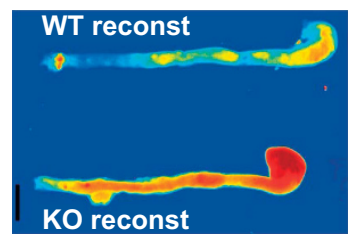

g
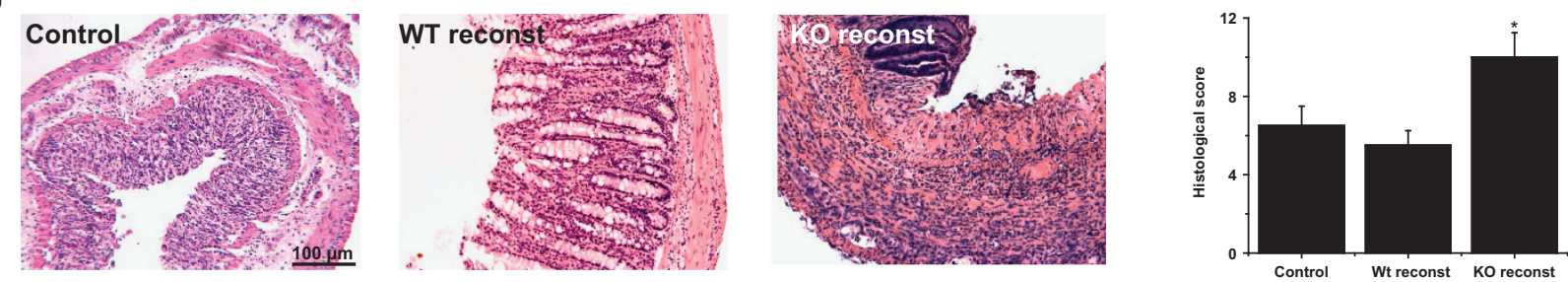

Figure 6 Enhanced DSS (2\%) colitis susceptibility of wild-type (WT) mice reconstituted with TRPM8-deficient macrophages. (a) Scheme of the macrophage adoptive transfer colitis experiments. In the treatment group, mice were repeatedly injected clodronate liposomes (CL) IP on day $-7,-5$, and -3 (control non-depleted WT mice received IV PBS liposomes). In parallel, bone marrow (BM)-derived mononuclear cells from wild-type and TRPM8 knockout mice were differentiated in vitro for 7 days into macrophages under the influence of M-CSF. Differentiated macrophages were transferred IV to the recipient mice on day 0 (control mice received IV PBS instead of macrophages); in parallel the DSS (2\%) drinking water regimen was started. To assure that some liposomes, which were possibly outlasting in the blood circulation, do not destroy a part of the transferred macrophage population, IV adoptive macrophage transfer was repeated on day 4 of the DSS colitis, 7 days after the last CL injection. Colitis was monitored by daily body weight measurement and microendoscopy as indicated in the flow chart. Detailed description of the different experimental groups: Control: non-depleted WT C57BL/6 mice receiving IP vehicle (PBS liposomes)-controls during the phase of depletion and receiving IV PBS in the phase of reconstitution. WT reconst: WT C57BL/6 mice receiving IP clodronate liposomes during the phase of depletion and receiving IV WT (TRPM8 ${ }^{+/+}$) macrophages in the phase of reconstitution. KO reconst: WT C57BL/6 mice receiving IP clodronate liposomes during the phase of depletion and receiving IV KO (TRPM8 ${ }^{-1-}$ ) macrophages in the phase of reconstitution. (b) Mice reconstituted with TRPM8-deficient macrophages (KO reconst) demonstrated higher weight loss during the course of DSS colitis compared with mice that received TRPM8 ${ }^{+/+}$macrophages (WT reconst) or non-depleted WT controls (control). Data are representative of three experiments, each group $n=8$ mice. ${ }^{\star} P<0.05,{ }^{\star \star} P<0.01$, analysis of variance followed by Fisher's LSD test. (c) Higher letality of mice during DSS colitis that received TRPM8-deficent macrophages compared with those receiving WT macrophages and non-depleted controls. ${ }^{*} P<0.05$, ${ }^{*} P<0.01$, analysis of variance followed by Fisher's LSD test compared with control. (d) Cytokine levels from colonic tissue isolated at the end of the experiment demonstrated decreased IL-10 and slightly increased TNF- $\alpha$ content in WT mice that were reconstituted with TRPM8 ${ }^{-1-}$ macrophages. (e) WT mice that were reconstituted with TRPM8 ${ }^{-1-}$ macrophages showed aggravated endoscopical colitis scores compared with mice that received TRPM8 ${ }^{+/+}$macrophages or controls. ${ }^{*} P<0.05$ Mann-Whitney U-test. Representative endoscopical photographs of the colon from the three different groups. (f) Representative ex vivo fluorescence image of reactive oxygen species (ROS) from the isolated murine colon (black scale $\mathrm{bar}=10 \mathrm{~mm}$ ) of WT mice receiving either TRPM8-deficient $(\mathrm{KO})$ or TRPM8 ${ }^{+/+}(\mathrm{WT})$ macrophages. The image was taken immediately after killing of mice at the end of the 8 day experiment. (g) Representative histological slides from the murine colon of the different groups at the end of experiment $(\times 20$ magnification, scale bar $100 \mu \mathrm{m}$ ). Histological score of the different DSS colitis groups. Data are representative of three experiments, each group $n=8$ mice. ${ }^{*} P<0.05$ Mann-Whitney $U$-test. 

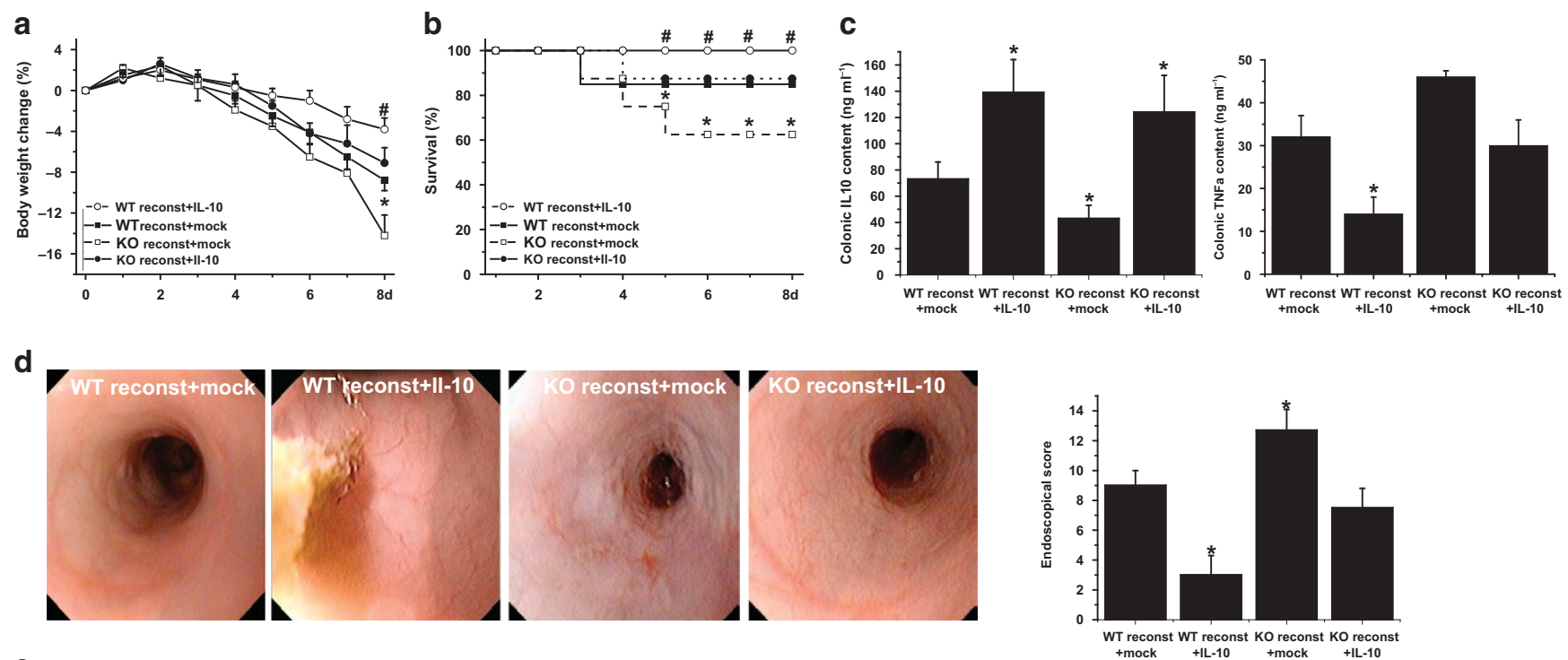

e
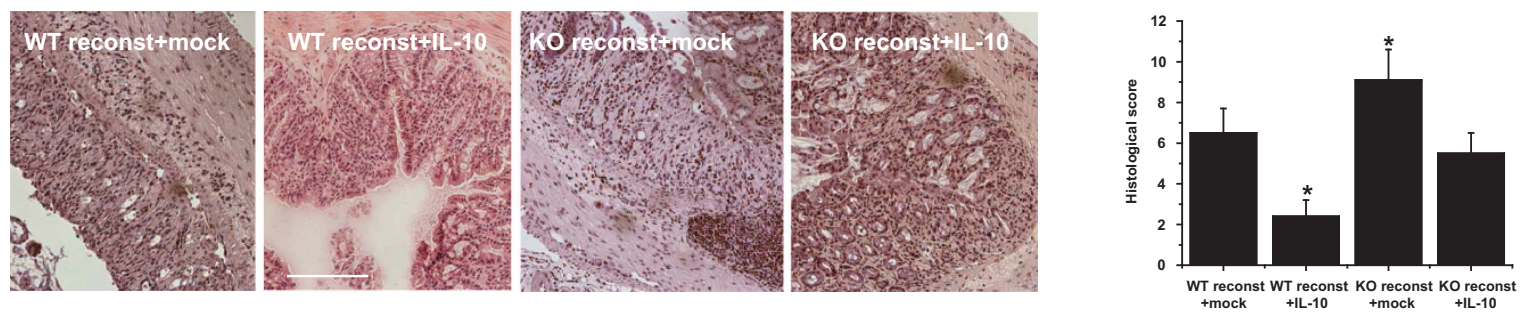

Figure 7 Systemic overexpression of IL-10 rescues the phenotype of DSS colitis aggravation induced by TRPM8-deficient macrophages. Detailed description of the different experimental groups: WT reconst + IL-10: CL-depleted WT C57BL/6 mice reconstituted with WT macrophages receiving IV IL-10 construct. KO reconst + IL-10: CL-depleted WT C57BL/6 mice reconstituted with TRPM8 ${ }^{-1-}$ macrophages receiving IV IL-10 construct. WT reconst + mock: CL-depleted WT C57BL/6 mice reconstituted with TRPM8 ${ }^{+/+}$macrophages receiving IV mock. KO reconst + mock: CL-depleted WT C57BL/ 6 mice reconstituted with TRPM $8^{-I-}$ macrophages receiving IV mock. (a) Mice receiving TRPM ${ }^{-1-}$ macrophages plus the IL-10 vector as well as mock-treated mice receiving TRPM $8^{+/+}$macrophages demonstrated less body weight loss and (b) a higher survival rate compared with mock-treated mice reconstituted with TRPM8 ${ }^{-1-}$ macrophages. Mice that were reconstituted with WT macrophages and received the IL-10 vector showed the best outcome. (c) Colonic cytokine content indicates reduction of IL-10 levels (left panel) in isolated colons from mock-treated mice reconstituted with TRPM $^{-1-}$ macrophages and slightly increased (n.s.) TNF- $\alpha$ levels (right panel). Mice that were reconstituted with WT macrophages and received the IL-10 construct showed significantly elevated colonic IL-10 and reduced TNF- $\alpha$ contents. (d) Representative endoscopic photographs and endoscopic score as well as $(\mathbf{e})$ representative histological slides $(\times 20$ magnification, scale bar $100 \mu \mathrm{m})$ from the mouse colon of the different groups with histological score. Data are representative of three experiments, each group $n=6$. $(\mathbf{a}, \mathbf{b})^{\sharp, \star} P<0.05,{ }^{\star \star} P<0.01$, analysis of variance followed by Fisher's LSD test. (c-e) ${ }^{\star} P<0.05$ Mann-Whitney $U$-test, each statistics in relation to WT reconst + mock.

function in the context of inflammation. In murine PM, the lack of TRPM8 activity led to a pro-inflammatory cytokine profile and impaired phagocytosis, whereas TRPM8 activation induced the opposite effects in vitro. In vivo, TRPM8 activation via repeated menthol enemas protected mice from experimental colitis. In contrast, global TRPM8 KO mice and mice reconstituted with TRPM8-deficient macrophages exhibited increased colitis susceptibility caused by a balance-shift of TNF- $\alpha$ and IL-10 secretion.

Previously, several TRP channels were ascribed important roles in macrophage function. TRPC3-deficient macrophages were found to exhibit constitutively reduced cation influx, increased apoptosis, and impaired efferocytosis. ${ }^{9}$ TRPC6 expression was observed in human alveolar macrophages and lung tissue macrophages, and TRPC6-like currents were identified in the latter cell type using whole-cell patch-clamp electrophysiology. Elevated TRPC6 mRNA expression in alveolar macrophages from patients with chronic obstructive pulmonary disease suggests a potential therapeutic target. ${ }^{10}$ Monocytes expressing TRPM2 exhibit an enhanced inflammatory capacity. ${ }^{23,24}$ Accordingly, TRPM2-deficient mice are highly susceptible to Listeria monocytogenes infection and resistant to DSS colitis, which is mediated by innate immune cells. ${ }^{23,24}$ TRPV2-specific short hairpin RNA inhibited LPS-induced TNF- $\alpha$ and IL-6 production in RAW 264.7 macrophages, and the LPS-induced increase in intracellular calcium levels was suggested to involve both TRPV2-mediated intracellular and extracellular $\mathrm{Ca}^{2+}$ mobilization. In murine PM, TRPV2 mediates particle binding and phagocytosis, and TRPV2-deficient mice exhibit substantially compromised host defense against bacterial infection (L. monocytogenes) due to suppressed bacterial clearance. ${ }^{11}$ Previously, TRPV4 expression was observed in murine alveolar macrophages, and the TRPV4 agonist $4 \alpha$-PDD induced calcium influx in alveolar macrophages from WT but not global TRPV4 null mutant animals. Moreover, TRPV4 ${ }^{+/+}$macrophages restored 
a

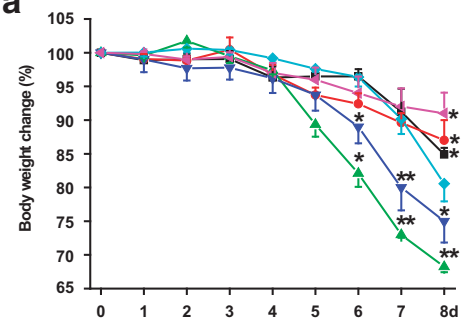

b

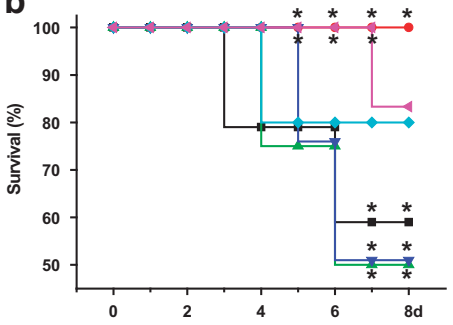

DSS colitis in

- - TRPM8 KO+WT reconst+vehicle enema

- - TRPM8 KO+WT reconst+menthol enema

- - TRPM8 KO mice+vehicle enema

- $\mathbf{-}$ - TRPM8 KO mice+menthol enema

- WT mice+vehicle enema

- - WT mice+menthol enema
C

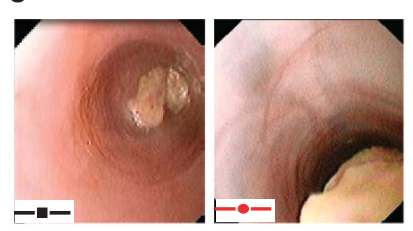

d

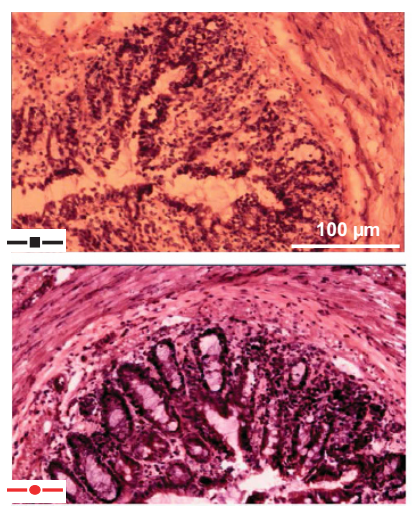

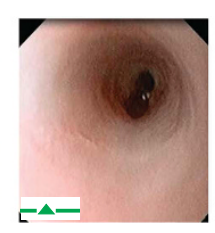
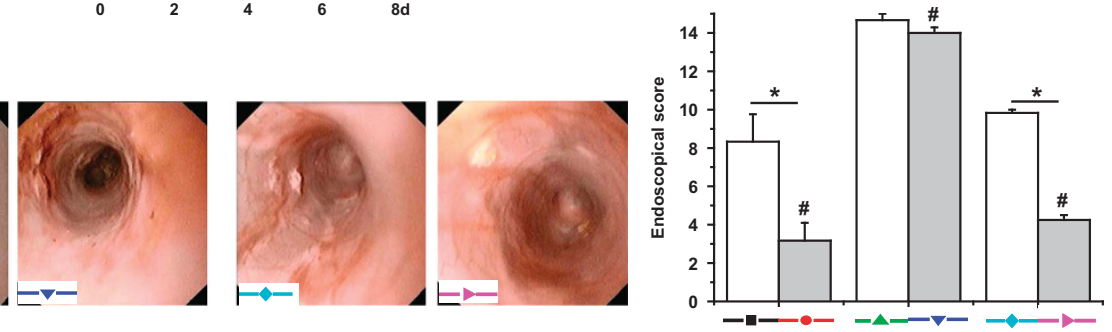
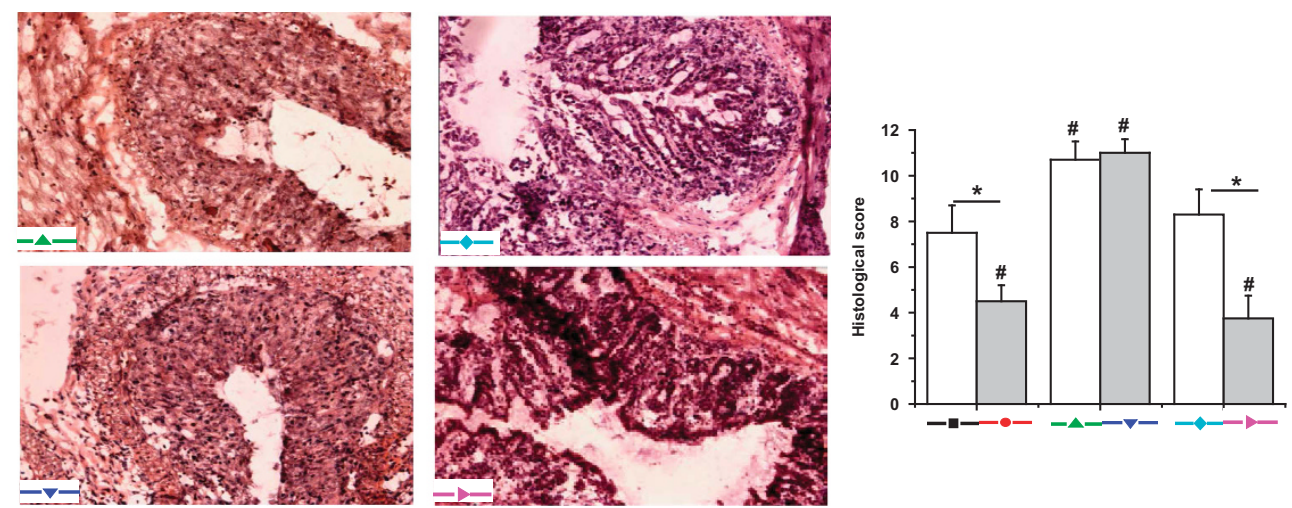

Figure 8 Menthol attenuates DSS colitis in mice that harbor WT macrophages independent of the TRPM8 status in other cells than macrophages. Detailed description of the different experimental groups: TRPM8 KO + WT reconst + vehicle enema: CL-depleted TRPM8 KO mice reconstituted with WT macrophages receiving vehicle enemas. TRPM8 KO + WT reconst + menthol enema: CL-depleted TRPM8 KO mice reconstituted with WT macrophages receiving menthol enemas. WT mice + menthol enema: undepleted WT mice receiving menthol enemas. WT mice + vehicle enema: undepleted WT mice receiving vehicle enemas. TRPM8 KO mice + vehicle enema: undepleted TRPM8 KO mice receiving vehicle enemas. TRPM8 KO mice + menthol enema: undepleted TRPM8 KO mice receiving menthol enemas. (a) Menthol was effective in the two groups of mice that harbor WT macrophages, which is mirrored by less body weight loss, (b) increased survival rates and an improved, (c) endoscopical, and (d) histological outcome. Pictures show representative endoscopic photographs and histological slides ( $\times 20$ magnification, scale bar $100 \mu \mathrm{m})$ from the mouse colon of the different groups. Data are representative of three experiments, each group $n=5-8$. (a) ${ }^{\star} P<0.05$, ${ }^{\star *} P<0.01$, analysis of variance followed by Fisher's LSD test compared with the WT mice + vehicle enema group (body weight and survival statistics); (c,d) ${ }^{\star} P<0.05$ Mann-Whitney $U$-test as indicated by horizontal lines. \# indicates significance compared with the WT mice + vehicle enema group.

the susceptibility of TRPV4 $4^{-/}$lungs to ventilator-induced mechanical injury. ${ }^{12}$

However, to date, only one report has used a patch-clamp analysis to suggest TRPM8-like function in a macrophage-like cell line (RAW 264.7). ${ }^{18}$ We confirmed TRPM8 immunoreactivity in RAW cells and provided evidence for TRPM8 expression in murine PM, BMDM and colonic LPMC. Moreover, we demonstrated that TRPM8 has a critical immunomodulatory role in macrophages. Direct inhibition of pro-inflammatory cytokine production by menthol was shown previously in human lymphocytes and other respiratory tract monocytes. ${ }^{17}$ In our study, TRPM8 activation induced the release of IL-10 but suppressed the release of TNF- $\alpha$ from PM in vitro, whereas TRPM8-deficient PM exhibited the opposite phenotype. Moreover, TRPM8 activation led to increased phagocytosis of zymosan particles in vitro, whereas
TRPM8-deficient PM exhibited defective phagocytosis of fluorescent beads in vivo. Using a more physiological model of bacterial phagocytosis (Citrobacter rodentium), we confirmed a deficit in the phagocytic capacity of TRPM8 KO macrophages. This fundamental role of TRPM8 in macrophage phagocytosis may have important implications for various bacterial infectious diseases, possibly leading to new antiinfective pharmaceuticals.

Previous studies of TRPM8 function with regard to the gastrointestinal tract have shown that peroral treatment with the TRPM8 agonist menthol induced gastroprotective activity through anti-apoptotic, anti-oxidant, and anti-inflammatory mechanisms that involved reductions in TNF- $\alpha$ and IL-6 expression and increased IL-10 expression in the gastric mucosa, ${ }^{25}$ in accordance with the menthol-induced changes in the in vitro macrophage cytokine pattern observed in our study. 
Moreover, eucalyptol (1,8-cineol) enemas were previously found to attenuate rat trinitrobenzene sulfonic acid (TNBS)induced colitis. ${ }^{19}$ A recent study demonstrated that systemic TRPM8 activation induced by icilin $\left(4 \mathrm{mg} \mathrm{kg}^{-1} \mathrm{IP}\right)$ attenuated acute DSS and TNBS colitis. ${ }^{5}$ In our study we preferred local application (enemas) and used a model of DSS-induced colitis in which the innate immune system has a more dominant role than in TNBS-induced colitis. ${ }^{22}$ In our hands, twice-daily topical TRPM8 activation via menthol enemas strongly attenuated DSS colitis. The TRPM8-mediated anti-inflammatory molecular mechanisms reported by Ramachandran et al. ${ }^{5}$ remained vague. As TRPM8 activation reduced the TRPV1mediated release of CGRP from peptidergic colonic sensory neurons in vitro, the authors had speculated that inhibited neurogenic inflammation may have been responsible for the observed effects. ${ }^{5}$ However, this latter mechanism appears questionable, as CGRP has a rather protective effect against colitis. ${ }^{6,26,27}$ In the earlier report, global TRPM8 KO mice with acute DSS (1\% and 2.5\%) and TNBS colitis inconsistently exhibited the same disease severity as WT mice, although the colonic CGRP levels were truly increased in TRPM8 KO mice. In contrast, a study by de Jong et al. ${ }^{3}$ found that global TRPM8 KO mice developed more severe acute (innate immune cell-mediated) DSS (2 and 1\%) colitis relative to WT mice. CGRP treatment reversed the hyperinflammatory phenotype of TRPM8 KO mice and insufficient release of neuronal CGRP into the tissue microenvironment led to dysregulation of DC function, which was previously shown to have a vital role in colitis development. ${ }^{3,28}$ We confirmed the increased susceptibility of TRPM8 KO mice to acute DSS (2\%) colitis relative to WT mice. To clarify whether changes in the release of colonic CGRP may be responsible for the therapeutic effects of menthol enemas, we measured neuropeptide release in isolated colons from healthy and colitic mice. Both, menthol and icilin inhibited (mechanically)-induced CGRP release in the healthy colon, but not in the inflamed colon, where the peptidergic sensory neurons were obviously either desensitized or depleted of neuropeptides. It remains speculative that the defective CGRP release in the inflamed colon is a result of the desensitization of TRP channels such as TRPA1, TRPV4 or exactly TRPM8 that we have previously shown to be important in mechanically induced nociception of the healthy murine colon. ${ }^{29}$ Noteworthy, the observed inhibition/reduction of CGRP release may also indicate a reduction in substance $\mathrm{P}$ release, another important neuropeptide crucially involved in colitis pathogenesis. ${ }^{6}$

The results of our study suggest that the physiologically relevant anti-inflammatory effects of TRPM8 activation are mediated on the level of macrophage functioning. In particular, the results of the adoptive macrophage transfer experiment in clodronate liposome-treated mice strongly suggest that TRPM8 acts as a direct immunomodulator in macrophages. Only mice that harbored WT macrophages were protected from DSS colitis by menthol enemas, which was independent of TRPM8 expression in non-macrophage tissue. TRPM8 activation attenuated the release of the pro-inflammatory cytokine
TNF- $\alpha$ and increased secretion of the immunoregulatory cytokine IL-10 from macrophages in vitro. IL-10 has been recognized as a key immunosuppressive cytokine, and polymorphisms in the IL-10 gene locus confer a risk of ulcerative colitis and Crohn's disease. ${ }^{1,30,31}$ Mice and humans deficient in either IL-10 or the IL-10 receptor exhibit severe intestinal inflammation. ${ }^{32}$ IL-10 regulates intestinal inflammation independently of T cell-specific IL-10 receptor signaling, supporting a role for IL-10 signaling in innate immunity. ${ }^{1}$ Our in vitro data from TRPM8-deficient PM shows that activation of TRPM8 as wells as the lack of this receptor subtype leads to a balance-shift of pro- and anti-inflammatory cytokines. In vivo this balanceshift of IL-10 and TNF- $\alpha$ production was only partly comprehensible. For example, exaggerated DSS colitis in mice reconstituted with TRPM8-deficient BMDM may be explained partly through their shift in cytokine levels including the demonstrated reduction of IL-10 content in the colonic tissue. At first sight, this appears to be in contrast with two recent reports that show that macrophage-derived IL-10 is dispensable for gut homeostasis; however, T reg-derived IL-10 and sensing of IL-10 via IL- 10 receptor by CX3CR ${ }^{\text {hi }}$ intestinal macrophages was critical. ${ }^{1,33}$ Macrophage-specific deletion of the IL-10 receptor (IL-10Ra) but not of Il-10 led to the development of spontaneous colitis. ${ }^{33}$ In our study, the reconstitution of macrophage-depleted mice with TRPM8deficient BMDM resulted in an exacerbated DSS colitis but not in spontaneous colitis. The induction of colitis by DSS initiates numerous pathological conditions that involve disintegration of the epithelial layer consecutive activation of various immune cells in the colonic mucosa. The fact that clodronate liposomes (CL)-depleted mice per se show strong attenuation of DSS colitis supports an essential role of macrophages in colitis development, here especially in the DSS model, and demonstrates that macrophages orchestrate that immunological reaction. However, in that experiment other immune cells compensated for IL-10 production, at least measured in the colonic content. Interestingly, a previous report demonstrated the crucial role of macrophages in the development of spontaneous chronic colitis in IL-10-deficient mice using microspheres containing dichloromethylene diphosphonate. ${ }^{34}$ Moreover, we demonstrate that systemic overexpression of IL10 dampens the aggravated colitis level to the level of control mice that were reconstituted with WT BMDM. A previous report already demonstrated the anti-inflammatory effect of systemic IL-10 treatment in murine colitis, ${ }^{35}$ which we confirm in our group of mice that were reconstituted with WT macrophages and received the IL-10 construct. Whether systemic treatment by overexpression of IL-10 really compensates for the deficiency of IL-10 production in TRPM8deficient macrophages in vivo remains unclear. It also remains unclear whether the phenotype of TRPM8 KO mice or that of WT mice reconstituted with TRPM8 KO macrophages is owing to potential downregulation of the IL-10Ra on macrophages rather than decreased IL-10 and increased TNF- $\alpha$ production. The reason that overexpression of IL-10 was less effective in mice with TRPM8 KO macrophages than in the ones with WT 
macrophages could finally be due to the relative loss of its binding sites. However, the degree of colitis suppression induced by systemic IL-10 overexpression was stronger in mice receiving WT macrophages, which indicates that the described changes in cytokine expression or motility in TRPM8-deficient mice may have intrinsic effects on colitis severity.

Still, the underlying mechanism by which TRPM8 ultimately controls macrophage function is not yet completely understood. In the present work we have only measured two key cytokines TNF- $\alpha$ and IL-10 and analyzed the phosphorylation of two important members of the MAP kinases family that are involved in the regulation of their expression. TRPM8 is a cation-selective ion channel with a preference for calcium-ion permeation. Several calcium-activated pathways have been described to be involved in the phosphorylation of ERK. The formation of calcium-calmodulin complexes and calciummediated activation of protein kinase C via RAF1 and MEK1/2 are only two mechanisms that may explain TRPM8-mediated upregulation of p-ERK. ${ }^{36,37}$ Moreover, we can only speculate about the mechanism that led to the absence of p-ERK in TRPM8-deficient PM. Probably, the lack of TRPM8 caused a balance-shift in calcium homeostasis, which obviously led to a defective phosphorylation of ERK, which is at least one of the possible mechanisms that resulted in the demonstrated balance-shift of IL-10 and TNF- $\alpha$ signaling in TRPM8deficient PM. The involvement of intracellular protein cascades downstream of TRPM8 in addition to the p38 and ERK pathways also seems very likely. Recently, a novel multi-protein signaling complex was identified; in TRPM8-expressing sensory neurons, the TRPM8 channel was shown to form a complex with the 5-HT1B receptor and phospholipase D1. ${ }^{38}$ This complex subsequently activated the type I phosphatidylinositol-4-phosphate 5-kinase to generate phosphatidylinositol 4,5-bisphosphate, commonly referred to as PIP2. Because PIP2 is a positive regulator of TRPM8, an inflammatory agent such as bradykinin, which activates a PLC-coupled receptor and leads to PIP2 hydrolysis, could potentially result in TRPM8 channel desensitization and subsequent pro-inflammatory effects on macrophage functioning, as indicated by our in vitro and in vivo data. The PLC-PIP2-TRPM8 pathway is of particular interest in terms of a physiological role for TRPM8, as to date it remains unclear which endogenous agonists act on TRPM8 under inflammatory conditions. Of note, many novel TRPM8 agonists were recently identified, including some isoxazole derivatives that form the basis for a number of drugs (e.g., COX-2 inhibitors) $;^{39}$ these may exert anti-inflammatory effects through TRPM8 activation, in addition to COX inhibition. Interestingly, our fluorometric measurements of PM and BMDM revealed distinct desensitization of the TRPM8-mediated calcium ion influx, which is typical of many TRP family ion channels and was previously described for TRPM8 in sensory neurons. The proposed mechanism is similar to that described above, namely that $\mathrm{Ca}^{2+}$ influx through TRPM8 activates a $\mathrm{Ca}^{2+}$-sensitive PLC isoform that in turn hydrolyses PIP2, and the depletion of this lipid limits TRPM8 channel activity. ${ }^{40,41}$ Dialysis of PIP2 through a whole-cell patch pipette inhibited the desensitization of menthol-induced currents in both expression systems and in dorsal root ganglion (DRG) neurons.

In summary, our findings identify TRPM8 as a potential target in inflammatory states generally involving macrophage function (e.g., microbial infections) and particularly including the treatment of inflammatory bowel diseases. Clinical trials employing enemas or oral preparations of TRPM8 agonists such as icilin, menthol, and eucalyptol should be straightforward, as no severe side effects have been reported with these agents. ${ }^{42}$ Furthermore, peppermint oil-based remedies are already used by patients with irritable bowel syndrome because of their analgesic, antispasmodic, and carminative effects; patients with inflammatory bowel disease may also benefit from these effects.

\section{METHODS}

For details, see the Supplementary Methods.

Animals. WT and TRPM8-mutant (B6.129P2-Trpm $8^{\mathrm{tm} 1 J u l} / \mathrm{J}$, the Jackson laboratory, Bar Harbor, ME $)^{43}$ C57BL/6 mice of both sexes were used at 8-12 weeks of age. All animal experiments were approved by the Animal Protection Authority, District Government Mittelfranken, Ansbach, Germany.

Isolation of murine PM and bone marrow-derived macrophages. For preparation and cell isolation details, see the Supplementary Methods. As previously described, the procedures assured that $>99 \%$ of the attached cells were $\mathrm{F} 4 / 80^{+}$macrophages. ${ }^{11}$

Phagocytosis assay. The investigator who quantified phagocytic activity in all groups was blinded to genotypes and treatment procedures. In vitro phagocytosis was assessed using zymosan particles or Citrobacter rodentium; in vivo, Fluoresbrite Microparticles $\left(0.5 \mu \mathrm{lg}^{-1}\right.$; Polysciences, Eppelheim, Germany) were used to assess phagocytosis.

Ratiometric $\left[\mathrm{Ca}^{2+}\right]_{\mathrm{i}}$ measurements. Ratiometric $\left[\mathrm{Ca}^{2+}\right]_{\mathrm{i}}$ measurements of cultured murine PM and BMDM using Fura-2 $\left(5 \mu \mathrm{moll}^{-1}\right)$ were essentially performed as described previously with regard to neurons. ${ }^{6}$

Induction of DSS colitis, monitoring, and pharmacological treatment. Colitis was induced by adding 2\% DSS (molecular weight: $36,000-50,000 \mathrm{kDa}$; MP Biomedicals, Illkirch, France) to the drinking water for 1 week as described previously. ${ }^{6}$ Endoscopic colitis scoring was performed as previously described, using a murine endoscopic index of colitis severity (endoscopical score) consisting of five parameters: stool consistency, mucosal surface granularity, visible fibrin exudate, vascular pattern changes, and edematous colonic wall thickening accompanied by a loss of transparency. ${ }^{44}$ Each parameter was graded from 0 points (none) to 3 points (severe).

In vivo depletion of macrophages, adoptive transfer, and reconstitution. This method was originally described by Van Rooijen and Sanders. ${ }^{45}$

In vivo overexpression of IL-10. An expression construct for sustained in vivo IL-10 expression was generated by cloning a complementary DNA (cDNA) fragment encoding murine full-length cDNA into the AAV virus as described previously. ${ }^{46} \mathrm{DNA}$ was isolated using Qiagen plasmid maxi kits, which include an endotoxin removal step. Subsequently, the DNA was treated with a MiraClean endotoxin removal kit (Mirus Bio, Madison, WI). For treatment, $10 \mu \mathrm{g}$ of construct were administered in Krebs-Ringer solution to each mouse via hydrodynamic tail vein injection, as described 
previously.${ }^{47}$ Control mice received a control plasmid not encoding for IL-10 (mock).

Detection of reactive oxygen species. The MAESTRO full-body fluorescence scanning system (INTAS, Göttingen, Germany) was used for in vivo multispectral fluorescence analysis, as described recently. ${ }^{43}$ ROS detection was performed using ROS Brite $700 \mathrm{~nm}$ dye according to the manufacturer's guidelines (AAT Bioquest, Sunnyvale, CA).

Data analysis. The number $(n)$ refers to the number of animals or cells studied. Calculations were performed using Statistica 7.0 (Statsoft, Tulsa, OK) and Origin 7.0 (OriginLab, Northampton, MA). The tests used are denoted in the text and/or figure legends.

SUPPLEMENTARY MATERIAL is linked to the online version of the paper at http://www.nature.com/mi

\section{ACKNOWLEDGMENTS}

We thank I. Izydorczyk, A. Kuhn, J. Schramm, B. Vogler, and S. Haux-Oertel for technical assistance (Institute of Physiology and Pathophysiology). The present work was performed in fulfillment of the requirements for obtaining the degree 'Dr rer. nat.' for M. Khalil. M.A.E. received support from the Deutsche Forschungsgemeinschaft (DFG EN 1060/2-1), Marohn-Stiftung, ELAN, IZKF, and Universitätsbund of the Friedrich-Alexander-Universität Erlangen-Nürnberg. P.W.R. was supported by the Federal Ministry of Edu. \& Res. (BMBF0315449C).

\section{AUTHOR CONTRIBUTION}

MK: acquisition of data; analysis and interpretation of data; writing of the manuscript. $A B, R L, S F$ : acquisition of data; analysis and interpretation of data. PWR, SW, CB, MFN: study concept and design; critical revision of the manuscript for important intellectual content. MAE: study concept and design; acquisition of data; analysis and interpretation of data; critical revision of the manuscript for important intellectual content; writing of the manuscript; statistical analysis; obtained funding; study supervision.

\section{DISCLOSURE}

The authors declare no conflict of interest.

c) 2016 Society for Mucosal Immunology

\section{REFERENCES}

1. Shouval, D.S. et al. Interleukin-10 receptor signaling in innate immune cells regulates mucosal immune tolerance and anti-inflammatory macrophage function. Immunity 40, 706-719 (2014).

2. Nilius, B. \& Owsianik, G. The transient receptor potential family of ion channels. Genome. Biol. 12, 218 (2011).

3. de Jong, P.R. et al. TRPM8 on mucosal sensory nerves regulates colitogenic responses by innate immune cells via CGRP. Mucosal Immunol. 8, 491-504 (2015).

4. D'Aldebert, E., Cenac, N. \& Rousset, P. Transient receptor potential vanilloid 4 activated inflammatory signals by intestinal epithelial cells and colitis in mice. Gastroenterology 140, 275-285 (2011).

5. Ramachandran, R. et al. TRPM8 activation attenuates inflammatory responses in mouse models of colitis. Proc. Natl. Acad. Sci. USA 110, 7476-7481 (2013).

6. Engel, M.A. et al. TRPA1 and substance P mediate colitis in mice. Gastroenterology 141, 1346-1358 (2011).

7. Caceres, A.I. et al. A sensory neuronal ion channel essential for airway inflammation and hyperreactivity in asthma. Proc. Natl. Acad. Sci. USA 106, 9099-9104 (2009).

8. Woolf, C.J. \& Ma, Q. Nociceptors-noxious stimulus detectors. Neuron 55, 353-364 (2007).

9. Tano, J.Y. et al. Impairment of survival signaling and efferocytosis in TRPC3-deficient macrophages. Biochem. Biophys. Res. Commun. 410, 643-647 (2011).
10. Finney-Hayward, T.K. et al. Expression of transient receptor potential C6 channels in human lung macrophages. Am. J. Respir. Cell Mol. Biol. 43, 296-304 (2010).

11. Link, T.M. et al. TRPV2 has a pivotal role in macrophage particle binding and phagocytosis. Nat. Immunol. 11, 232-239 (2010).

12. Hamanaka, K. et al. TRPV 4 channels augment macrophage activation and ventilator-induced lung injury. Am. J. Physiol. Lung Cell Mol. Physiol. 299, 353-362 (2010).

13. Tsavaler, L. et al. Trp-p8, a novel prostate-specific gene, is up-regulated in prostate cancer and other malignancies and shares high homology with transient receptor potential calcium channel proteins. Cancer Res. 61, 3760-3769 (2001).

14. McKemy, D.D., Neuhausser, W.M. \& Julius, D. Identification of a cold receptor reveals a general role for TRP channels in thermosensation. Nature 416, 52-58 (2002).

15. Peier, A.M. et al. ATRP channel that senses cold stimuli and menthol. Cell 108, 705-715 (2002).

16. Zhao, C. et al. 1,8-cineol attenuates LPS-induced acute pulmonary inflammation in mice. Inflammation 37, 566-572 (2014).

17. Juergens, U.R. et al. Inhibitory activity of 1,8 -cineol (eucalyptol) on cytokine production in cultured human lymphocytes and monocytes. Pulm. Pharmacol. Ther. 17, 281-287 (2004).

18. Wu, S.N., Wu, P.Y. \& Tsai, M.L. Characterization of TRPM8-like channels activated by the cooling agent icilin in the macrophage cell line RAW 264.7. J. Membr. Biol. 241, 11-20 (2011).

19. Santos, F.A. et al. 1,8-cineole (eucalyptol), a monoterpene oxide attenuates the colonic damage in rats on acute TNBS-colitis. Food Chem. Toxicol. 42, 579-584 (2004).

20. Yang, Y. et al. Functional roles of p38 mitogen-activated protein kinase in macrophage-mediated inflammatory responses. Mediators Inflamm. 2014, 352371 (2014).

21. Gabryšová, L. et al. The regulation of IL-10 expression. Curr. Top. Microbiol. Immunol. 380, 157-190 (2014).

22. Wirtz, S. et al. Chemically induced mouse models of intestinal inflammation. Nat Protoc 2, 541-546 (2007).

23. Yamamoto, S. et al. TRPM2-mediated Ca2 + influx induces chemokine production in monocytes that aggravates inflammatory neutrophil infiltration. Nat. Med. 14, 738-747 (2008).

24. Knowles, H. et al. Transient Receptor Potential Melastatin 2 (TRPM2) ion channel is required for innate immunity against Listeria monocytogenes. Proc. Natl. Acad. Sci. USA 108, 11578-11583 (2011).

25. Rozza, A.L. et al. The gastroprotective effect of menthol: involvement of anti-apoptotic, antioxidant and anti-inflammatory activities. PLoS One $\mathbf{9}$, e86686 (2014).

26. Mazelin, L. et al. Vagally dependent protective action of calcitonin generelated peptide on colitis. Peptides 20, 1367-1374 (1999).

27. Reinshagen, M. et al. Calcitonin gene-related peptide mediates the protective effect of sensory nerves in a model of colonic injury. $J$. Pharmacol. Exp. Ther. 286, 657-661 (1998).

28. Abe, K. et al. Conventional dendritic cells regulate the outcome of colonic inflammation independently of T cells. Proc. Natl. Acad. Sci. USA 104, 17022-17027 (2007).

29. Mueller-Tribbensee, S.M. et al. Differential contribution of TRPA1, TRPV4 and TRPM8 to colonic nociception in mice. PLoS One 10, e0128242 (2015).

30. Franke, A. et al. Sequence variants in IL10, ARPC2 and multiple other loci contribute to ulcerative colitis susceptibility. Nat. Genet. 40, 1319-1323 (2008).

31. Doecke, J.D. et al. Genetic susceptibility in IBD: overlap between ulcerative colitis and Crohn's disease. Inflamm. Bowel. Dis. 19, 240-245 (2013).

32. Mantovani, A. \& Marchesi, F. IL-10 and macrophages orchestrate gut homeostasis. Immunity 40, 637-639 (2014).

33. Zigmond, E. et al. Macrophage-restricted interleukin-10 receptor deficiency, but not IL-10 deficiency, causes severe spontaneous colitis. Immunity 40, 720-733 (2014).

34. Watanabe, N. et al. Elimination of local macrophages in intestine prevents chronic colitis in interleukin-10-deficient mice. Dig. Dis. Sci. 48, 408-414 (2003).

35. Tomoyose, M. et al. Role of interleukin-10 in a murine model of dextran sulfate sodium-induced colitis. Scand. J. Gastroenterol. 33, 435-440 (1998). 
36. Saraiva, M. \& O'Garra, A. The regulation of IL-10 production by immune cells. Nat. Rev. Immunol. 10, 170-181 (2010).

37. Kelly, E.K., Wang, L. \& Ivashkiv, L.B. Calcium-activated pathways and oxidative burst mediate zymosan-induced signaling and IL-10 production in human macrophages. J. Immunol. 184, 5545-5552 (2010).

38. Vinuela-Fernandez, I. et al. The TRPM8 channel forms a complex with the 5-HT1B receptor and phospholipase D that amplifies its reversal of pain hypersensitivity. Neuropharmacology 79, 136-151 (2013).

39. Ostacolo, C. et al. Isoxazole derivatives as potent transient receptor potential melastatin type 8 (TRPM8) agonists. Eur. J. Med. Chem. 69, 659-669 (2013).

40. Rohács, T. et al. PI(4,5)P2 regulates the activation and desensitization of TRPM8 channels through the TRP domain. Nat. Neurosci. 8, 626-634 (2005).

41. Daniels, R.L., Takashima, Y. \& McKemy, D.D. Activity of the neuronal cold sensor TRPM8 is regulated by phospholipase $\mathrm{C}$ via the phospholipid phosphoinositol 4,5-bisphosphate. J. Biol. Chem. 284, 1570-1582 (2009).
42. Rahimi, R. \& Abdollahi, M. Herbal medicines for the management of irritable bowel syndrome: a comprehensive review. World J. Gastroenterol. 18, 589-600 (2012).

43. Foersch, S., Kiesslich, R. \& Waldner, M.J. Molecular imaging of VEGF in gastrointestinal cancer in vivo using confocal laser endomicroscopy. Gut 59, 1046-1055 (2010).

44. Becker, C., Fantini, M.C. \& Neurath, M.F. High resolution colonoscopy in live mice. Nat. Protoc. 1, 2900-2904 (2006).

45. Van Rooijen, N. \& Sanders, A. Liposome mediated depletion of macrophages: mechanism of action, preparation of liposomes and applications. J. Immunol. Methods 174, 83-93 (1994).

46. McHedlidze, T. et al. Interleukin-33-dependent innate lymphoid cells mediate hepatic fibrosis. Immunity 39, 357-371 (2013).

47. Liu, X.L., Clark, K.R. \& Johnson, P.R. Production of recombinant adenoassociated virus vectors using a packaging cell line and a hybrid recombinant adenovirus. Gene. Ther. 6, 293-299 (1999). 